\title{
31
}

\section{Mixed finite element for stationary flow of a mixture with barodiffusion}

\author{
Piotr Krzyżanowski \\ Warsaw University \\ Institute of Applied Mathematics, ul. Banacha 2, 02-097 Warszawa, \\ Poland \\ E-mail: Piotr.Krzyzanowski@appli.mimuw.edu.pl
}

\begin{abstract}
We analyse a mixed finite element discretization of a system of equations describing the stationary, isothermic flow of a mixture with nonlinear barodiffusion. Using Taylor-Hood elements for velocity and pressure, and linear elements for concentration, we provide results on existence, uniqueness and approximation for derived discrete problem.
\end{abstract}

\section{Keywords}

Mixture flow, Stokes equations, barodiffusion, mixed finite element

\section{INTRODUCTION}

The stationary isothermic flow of a mixture of two incompressible viscous fluids with diffusion is described by the boundary value problem (Petrosyan, 1984), (Lukaszewicz, 1991):

$-\nu \Delta u+(u \cdot \nabla) u+\nabla p=f+c g$ in $\Omega$,

$\operatorname{div} u=0$ in $\Omega$,

$-\operatorname{div}(D(c) \nabla c)+u \cdot \nabla c=\operatorname{div}(K(c) \nabla p)$ in $\Omega$,

$u=u_{0}$ on $\partial \Omega$,

$c=c_{0}$ on $\partial \Omega$.

Here, $\Omega$ denotes a bounded open subset in $R^{3}$ or $R^{2}$. The unknowns are: the mean mass velocity vector $u$, the pressure $p$ in the mixture and the first component concentration $c$. The vectors $f, g$ denote external forces acting on the components of the mixture, $D$ and $K$ denote diffusion and barodiffusion coefficients, dependent on the concentration $c$. We assume that the viscosity $\nu$ is positive and constant. The boundary conditions on $u$ and $c$ are imposed by the trace of given functions $u_{0}, c_{0}$, defined on entire $\Omega$.

The above system of PDEs appears, for example, in mathematical models of the flow of some suspensions, such as blood (Popel et al., 1974). Existence and uniqueness of weak 
solutions to (1) - (5) has been investigated in (Lukaszewicz, 1991). This system is a basis for analysis of more advanced models of suspensions (e.g. (Krzyżanowski, 1994), where the micropolar fluid mixture is considered).

In case when the velocity is relatively small, it is physically reasonable to skip the nonlinear term in the Naver - Stokes equations, obtaining the following system:

$-\nu \Delta u+\nabla p=f+c g$ in $\Omega$

$\operatorname{div} u=0$ in $\Omega$,

$-\operatorname{div}(D(c) \nabla c)+u \cdot \nabla c=\operatorname{div}(K(c) \nabla p)$ in $\Omega$,

$u=u_{0}$ on $\partial \Omega$,

$c=c_{0}$ on $\partial \Omega$.

In this paper we present an analysis of finite element approximation of the solution of system (6) - (10), with additional assumption that the diffusion coefficient $D$ is a positive constant. For simplicity, we also assume homogeneous boundary condition on $u$.

Throughout the paper we assume, unless otherwise stated, that $\Omega$ is a bounded polygon in $R^{2}$ or a bounded polyhedron in $R^{3}$.

\subsection{Notation}

We shall use several function spaces, which properties are described, for example, in (Adams, 1975). By $W^{k, p}(\Omega)$ we shall denote the usual Sobolev spaces, identifying $W^{0, p}(\Omega)$ with the $L^{p}(\Omega)$ space of measurable functions with their $p$-th power Lebesgue integrable. The standard norm in $W^{k, p}$ shall be denoted by $\|\cdot\|_{k, p}$, while the seminorm - by $|\cdot|_{k, p}$. For the space $W^{k, 2}(\Omega)$ we shall use a symbol $H^{k}(\Omega)$, and the norm in that space we shall abbreviate as $\|\cdot\|_{k}$.

By $H_{0}^{1}(\Omega)$ we shall understand the subspace of $H^{1}(\Omega)$ of functions with their trace on $\partial \Omega$ equal to zero. By $L_{0}^{2}(\Omega)$ we denote the subspace of $L^{2}(\Omega)$, defined as

$L_{0}^{2}(\Omega)=\left\{w \in L^{2}(\Omega): \int_{\Omega} w=0\right\}$.

We denote the inner product in $L^{2}(\Omega)$ by brackets:

$(u, v):=\int_{\Omega} u v d x$

for any $u, v \in L^{2}(\Omega)$. Following (Temam, 1979), we also introduce a trilinear form

$b(u, v, w):=\frac{1}{2}((u \cdot \nabla v, w)-(u \cdot \nabla w, v))$

for any $u, v, w \in H^{1}(\Omega)$. This continuous form is by definition antisymmetric with respect to the last two arguments (which reflects the antisymmetry of $(u \cdot \nabla v, w)$ on the solution $u$ of $(6)-(10))$. In particular, we have

$b(u, v, v)=0$. 
By symbol "Const" we denote generic constant, independent of $h$, which, where necessary, we shall distinguish by subscripts.

Where there is no risk of confusion, we shall write $W^{k, p}, H^{k}, H_{0}^{1}, L_{0}^{2}$ instead of $W^{k, p}(\Omega)$, $H^{k}(\Omega), H_{0}^{1}(\Omega), L_{0}^{2}(\Omega)$.

\subsection{General assumptions.}

We shall assume there holds the following regularity condition:

(R1) For every $f \in L^{2}(\Omega)$ the weak solution $(u, p)$ of Stokes equation

$$
\begin{aligned}
-\Delta u+\nabla p=f & \text { in } \Omega, \\
\operatorname{div} u=0 \quad \text { in } \quad &
\end{aligned}
$$

with homogeneous Dirichlet boundary condition on $u$, and with $\int_{\Omega} p=0$, belongs to $\left(H^{2}(\Omega) \cap H_{0}^{1}(\Omega)\right) \times H^{1}(\Omega)$ and

$\|u\|_{2}+\|p\|_{1} \leq$ Const $\|f\|_{0}$.

for some Const independent of $f$.

For example, if $\Omega \subset R^{2}$ is a convex polygon, then assumption (R1) holds (Kellogg and Osborn, 1976).

We shall make the following assumptions on the data (see also (Lukaszewicz, 1991)):

(A1) $f, g \in L^{3}$;

(A2) $c_{0} \in H^{2}$ and $0 \leq c_{0}(x) \leq 1$ for $x \in \partial \Omega$;

(A3) $K: R \rightarrow R$ is Lipschitz continuous function:

$$
|K(s)-K(t)| \leq L_{K}|s-t|, \quad \forall t, s \in R
$$

and such that $K(s) \equiv 0$ for $s \notin(0,1)$.

\subsection{Discrete problem}

We shall work with the following function spaces:

$$
\begin{aligned}
& V:=H_{0}^{1}(\Omega)^{d}, \text { where } d=2,3 \text { is the dimension of } \Omega \subset R^{d}, \\
& W:=L_{0}^{2}(\Omega), \\
& X:=H_{0}^{1}(\Omega), \\
& X\left(c_{0}\right):=X+c_{0} .
\end{aligned}
$$

For homogeneous Dirichlet boundary condition on $u$, the variational formulation of the simplified problem (6) - (10) is as follows: 
Problem 1 Find $(u, p, c) \in V \times\left(W \cap H^{1}\right) \times X\left(c_{0}\right)$, such that

$\nu(\nabla u, \nabla v)-(p, \operatorname{div} v)=(f+c g, v) \quad \forall v \in V$,

$(\operatorname{div} u, w)=0 \quad \forall w \in W$,

$D(\nabla c, \nabla \xi)+b(u, c, \xi)=-(K(c) \nabla p, \nabla \xi) \quad \forall \xi \in X$.

This problem has a unique solution for sufficiently small and regular data in regular domains, and the proof is similar to that in (Lukaszewicz, 1991), where full problem (1) $-(5)$ is considered.

We approximate Problem 1 in finite dimensional subspaces $V_{h} \subset V, W_{h} \subset W, X_{h} \subset X$, $X_{h}\left(\tilde{c_{0}}\right):=X_{h}+\tilde{c_{0}}$, where $\tilde{c_{0}}$ is a (finite element) approximation of the boundary condition $c_{0}$, using mixed method:

Problem 2 Find $\left(u_{h}, p_{h}, c_{h}\right) \in V_{h} \times W_{h} \times X_{h}\left(\tilde{c_{0}}\right)$, such that

$$
\begin{aligned}
& \nu\left(\nabla u_{h}, \nabla v_{h}\right)-\left(p_{h}, \operatorname{div} v_{h}\right)=\left(f+c_{h} g, v_{h}\right) \quad \forall v_{h} \in V_{h}, \\
& \left(d i v u_{h}, w_{h}\right)=0 \quad \forall w_{h} \in W_{h}, \\
& D\left(\nabla c_{h}, \nabla \xi_{h}\right)+b\left(u_{h}, c_{h}, \xi_{h}\right)=-\left(K\left(c_{h}\right) \nabla p_{h}, \nabla \xi_{h}\right) \quad \forall \xi_{h} \in X_{h} .
\end{aligned}
$$

\subsection{Finite element assumptions.}

In our analysis we shall assume that the finite dimensional spaces $V_{h}, W_{h}, X_{h}$ are specific finite element spaces. We cover $\bar{\Omega}$ with a quasi-uniform, shape regular triangulation (Ciarlet, 1991) $\mathcal{T}_{h}$, dividing $\bar{\Omega}$ into triangles $K$ (or tetrahedra in three dimensional case)

$\bar{\Omega}=\bigcup_{K \in \mathcal{T}_{h}} K$

so that any $K \in \mathcal{T}_{h}$ has at least one vertex not on $\partial \Omega$ (Bercovier and Pironneau, 1979). The mesh parameter $h$ is defined as

$h=\max _{K \in \mathcal{T}_{h}} \operatorname{diam} K$.

Let $P_{j}(K)$ denote the space of polynomials of degree not greater than $j$ on single triangle $K \in \mathcal{T}_{h}$. We define the finite element spaces $V_{h}, W_{h}, X_{h}$ as follows.

For approximation of the velocity and pressure we use the Taylor - Hood finite elements (Brezzi and Fortin, 1991),

$V_{h}=\left\{v \in V \cap C(\bar{\Omega}):\left.v\right|_{K} \in P_{2}(K) \quad \forall K \in \mathcal{T}_{h}\right\}$

and

$W_{h}=\left\{w \in W \cap C(\bar{\Omega}):\left.w\right|_{K} \in P_{1}(K) \quad \forall K \in \mathcal{T}_{h}\right\}$.

Continuous finite element approximation of the pressure is necessary in our case, due to the $\nabla p_{h}$ term in (17). 
As concerns the space in which we approximate the concentration $c$, we shall consider $X_{h}$ consisting of linear elements, i.e.

$$
X_{h}=\left\{\xi \in X \cap C(\bar{\Omega}):\left.\xi\right|_{K} \in P_{1}(K) \quad \forall K \in \mathcal{T}_{h}\right\} .
$$

Properties of the above finite element spaces may be found in, for example, (Ciarlet, 1991).

We choose the finite element approximation $\tilde{c_{0}} \in C(\bar{\Omega})$ of the boundary condition $c_{0}$ so that $\left.\tilde{c_{0}}\right|_{K} \in P_{1}(K)$ for all $K \in \mathcal{T}_{h}$ and such that in the nodal points $x$ of triangulation $\mathcal{T}_{h}$ we have

$\tilde{c_{0}}(x)= \begin{cases}c_{0}(x) & \text { if } x \in \partial \Omega, \\ 0 & \text { otherwise. }\end{cases}$

\subsection{Main result}

Theorem 1 Suppose Problem 1 admits a solution $(u, p, c) \in\left(H^{3} \cap V\right) \times\left(H^{2} \cap W\right) \times$ $\left(H^{2} \cap X\left(\tilde{c}_{0}\right)\right)$ satisfying $\max \left\{\|u\|_{3},\|p\|_{2},\|c\|_{2}\right\} \leq$ Const $_{1}$. Under assumptions from paragraphs 1.2 and 1.4, there exist $H>0$ and some polynomial $P: R^{6} \rightarrow R$, with positive coefficients and with property $P(0,0,0,0,0,0)=0$, such that if the data of the problem satisfy condition

$P\left(D^{-1}, \nu^{-1},|K|_{\infty},\|f\|_{0,3},\|g\|_{0,3},\left\|c_{0}\right\|_{1}\right) \leq 1$,

then for any $0<h<H$ there exists a unique solution $\left(u_{h}, p_{h}, c_{h}\right)$ of Problem 2, which satisfies the following error estimate:

$\left\|u-u_{h}\right\|_{1}+\left\|p-p_{h}\right\|_{1}+\left\|c-c_{h}\right\|_{1}=O(h)$.

Let us comment on this theorem. The coefficients of polynomial $P$ depend only on $\Omega$, Const $_{1}$ and $H$. Since $P$ vanishes at $(0,0,0,0,0,0)$, then, by continuity, condition $(20)$ is satisfied for sufficiently small values of $D^{-1}, \nu^{-1},|K|_{\infty},\|f\|_{0,3},\|g\|_{0,3},\left\|c_{0}\right\|_{1}$. Hence, (20) is kind of small data requirement. This condition, evidently, might be replaced by simpler expression, like $D^{-1}+\nu^{-1}+|K|_{\infty}+\|f\|_{0,3}+\|g\|_{0,3}+\left\|c_{0}\right\|_{1} \leq$ Const $_{2}$, but polynomial condition is more descriptive, as it contains information that "smallness" of the data is relative one to another. Similar polynomial condition appears within uniqueness theorem for Problem 1 (Lukaszewicz, 1991), but, apparently, is weaker than derived here.

In our case we explicitly restrict ourselves only to the class of solutions which are uniformly bounded by Const ${ }_{1}$. However, if the domain boundary was smoother, then the assumption $\max \left\{\|u\|_{3},\|p\|_{2},\|c\|_{2}\right\} \leq$ Const $_{1}$ could have been replaced by "small data" requirement again. This follows from continuous dependence on data in above norms (Lukaszewicz, 1991).

The remaining of the paper is devoted to the proof of Theorem 1, and is organized as follows. In the next section, we briefly discuss a linearization of Problem 2, which decouples the system into two independent ones. Then in Section 3 we outline the proof of the existence statement of Theorem 1, based on Brouwer's fixed point theorem. Section 4 contains a scheme of the proof of the approximation part of Theorem 1, while in Section 5 we sketch the proof of the uniqueness result. 


\section{LINEARIZED DISCRETE PROBLEM}

Let us introduce an auxiliary linear problem:

Problem 3 Given $c_{h}^{*} \in X_{h}\left(\tilde{c_{0}}\right)$, find $\left(u_{h}, p_{h}, c_{h}\right) \in V_{h} \times W_{h} \times X_{h}\left(\tilde{c_{0}}\right)$, such that

$\nu\left(\nabla u_{h}, \nabla v_{h}\right)-\left(p_{h}, \operatorname{div} v_{h}\right)=\left(f+c_{h}^{*} g, v_{h}\right) \quad \forall v_{h} \in V_{h}$,

$\left(\operatorname{div} u_{h}, w_{h}\right)=0 \quad \forall w_{h} \in W_{h}$,

$D\left(\nabla c_{h}, \nabla \xi_{h}\right)+b\left(u_{h}, c_{h}, \xi_{h}\right)=-\left(K\left(c_{h}^{*}\right) \nabla p_{h}, \nabla \xi_{h}\right) \quad \forall \xi_{h} \in X_{h}$.

This problem may be seen as a linearization scheme for Problem 2, and may be used as a basis for an algorithm for iterative solution of Problem 2, with Stokes and diffusion equations decoupled. We shall use this linearization for the proof of existence and uniqueness of Problem 2.

Lemma 2 There exists exactly one solution $\left(u_{h}, p_{h}, c_{h}\right) \in V_{h} \times W_{h} \times X_{h}\left(\tilde{c_{0}}\right)$ of Problem 3. Moreover,

$D\left\|c_{h}\right\|_{1} \leq 2 D\left\|\tilde{c_{0}}\right\|_{1}+|K|_{\infty}\left\|\nabla p_{h}\right\|_{0}+$ Const $\left\|u_{h}\right\|_{1}\left\|\tilde{c_{0}}\right\|_{1}$.

Proof. The existence follows from (Girault and Raviart, 1986) and (Ciarlet, 1991). Taking $\xi=c-c_{0}$ in (24), we easily get (25).

\section{EXISTENCE OF SOLUTIONS TO DISCRETE PROBLEM}

Let us introduce a mapping $\Phi: X_{h} \rightarrow X_{h}$, defined as

$\Phi\left(c_{h}^{*}-\tilde{c_{0}}\right)=c_{h}-\tilde{c_{0}}$,

where $c_{h} \in X_{h}\left(\tilde{c_{0}}\right)$ is the solution of Problem 3 for given $c_{h}^{*} \in X_{h}\left(\tilde{c_{0}}\right)$. According to Lemma 2, this mapping is well defined.

First, we show that $\Phi$ is continuous. To this end, we consider the difference $\Phi\left(c_{1}^{*}-\tilde{c_{0}}\right)-$ $\Phi\left(c_{2}^{*}-\tilde{c}_{0}\right)$ for arbitrary $c_{1}^{*}, c_{2}^{*} \in X_{h}\left(\tilde{c_{0}}\right)$. By definition, this difference is equal to $c_{1}-c_{2}$, where $c_{1}, c_{2}$ are the solutions of Problem 3 with given $c_{1}^{*}, c_{2}^{*}$, respectively. Subtracting equations (24) we observe that $\bar{c}=c_{1}-c_{2}$ satisfies

$D(\nabla \bar{c}, \nabla \xi)+b\left(u_{1}, c_{1}, \xi\right)-b\left(u_{2}, c_{2}, \xi\right)=\left(K\left(c_{1}^{*}\right) \nabla p_{1}-K\left(c_{2}^{*}\right) \nabla p_{2}, \nabla \xi\right)$

for every $\xi \in X_{h}$. Taking $\xi=\bar{c}$ we obtain, due to (11) and imbedding $H^{1} \hookrightarrow L^{6}$,

$D|\bar{c}|_{1}^{2} \leq\left|b\left(u_{1}-u_{2}, c_{1}, \bar{c}\right)\right|+|\bar{c}|_{1} \operatorname{Const}\left(\left.L_{K}|| \nabla p_{2}\left|\left\|_{0,3}|| c_{1}^{*}-c_{2}^{*}\right\|_{1}+\right| K\right|_{\infty}\left\|p_{1}-p_{2}\right\|_{1}\right)$

Using inverse inequalities (Ciarlet, 1991) and estimates on solutions of discrete Stokes equations (Girault and Raviart, 1986), we obtain

$D\|\bar{c}\| \leq M\left\|c_{1}^{*}-c_{2}^{*}\right\|_{1}$, 
with constant $M=M\left(h, f, g, \tilde{c_{0}}, \nu, K, c_{1}^{*}\right)$, whence the continuity of $\Phi$. Next, we show that, for sufficiently small data (in the sense of Theorem 1), there exists a ball $\mathcal{M} \subset X_{h}$ such that $\Phi(\mathcal{M}) \subset \mathcal{M}$ and the diameter of $\mathcal{M}$ is independent of $h$.

We estimate $\left\|\Phi\left(c_{h}^{*}-\tilde{c_{0}}\right)\right\|_{1}$ in terms of $\left\|c_{h}^{*}-\tilde{c_{0}}\right\|_{1}$, obtaining

$\left\|\Phi\left(c_{h}^{*}-\tilde{c_{0}}\right)\right\|_{1} \leq A \cdot\left\|c_{h}^{*}-\tilde{c_{0}}\right\|_{1}+B$

where

$$
\begin{aligned}
& A=\frac{\text { Const }_{3}}{D}\left(\frac{\left\|\tilde{c_{0}}\right\|_{1}}{\nu}+|K|_{\infty}\right)\|g\|_{0,3}, \\
& B=\frac{\text { Const }}{D}\left(\frac{\left\|\tilde{c_{0}}\right\|_{1}}{\nu}+|K|_{\infty}\right)\|f\|_{0,3}+(A+1)\left\|\tilde{c_{0}}\right\|_{1} .
\end{aligned}
$$

Now, we take any positive real $A_{0}$ such that $A_{0}<1$. We define polynomial $P_{1}$ as

$P_{1}\left(x_{1}, x_{2}, \ldots, x_{6}\right)=\frac{\text { Const }_{3}}{A_{0}} x_{1} x_{5}\left(x_{2} x_{6}+x_{3}\right)$.

Suppose $P_{1}\left(D^{-1}, \nu^{-1},|K|_{\infty},\|f\|_{0,3},\|g\|_{0,3},\left\|c_{0}\right\|_{1}\right) \leq 1$, that is, the data are small enough in the sense of Theorem 1 . Then there exists $r>0$, such that $\Phi$ maps the ball $\mathcal{M}=$ $\left\{\xi \in X_{h}:\|\xi\|_{1} \leq r\right\}$ into itself. Let us stress that this invariant ball is independent of the mesh parameter $h$, so Brouwer's fixed point theorem yields the existence of solutions of Problem 2 for any value of $h$.

\section{ERROR ESTIMATE}

In this section we outline the derivation of estimate (21). We shall assume that Problem 1 admits solution $(u, p, c) \in\left(H^{3} \cap V\right) \times\left(H^{2} \cap W\right) \times\left(H^{2} \cap X\left(\tilde{c}_{0}\right)\right)$. Without loss of generality we can assume that the boundary condition on $c$ is represented exactly by $\tilde{c}_{0}$, since the solutions of Problem 1 depend continuously (Lukaszewicz, 1991) on $\tilde{c_{0}}$, and $\tilde{c_{0}}$ approximates $c_{0}$ on the boundary in $H^{1 / 2}$-norm with order $O(h)$.

Suppose there exists a solution $\left(u_{h}, p_{h}, c_{h}\right)$ of Problem 2. Using standard procedures and approximation results on discrete solutions of Stokes equations (Girault and Raviart, 1986), (Bercovier and Pironneau, 1979) we obtain

$$
\begin{aligned}
& \left\|u-u_{h}\right\|_{1} \leq O\left(h^{2}\right)+\frac{\text { Const }}{\nu}\|g\|_{0,3}\left\|c-c_{h}\right\|_{0,6} \\
& \left\|p-p_{h}\right\|_{1} \leq O(h)+\text { Const }\|g\|_{0,3}\left\|c-c_{h}\right\|_{0,6}
\end{aligned}
$$

and

$$
\begin{aligned}
D\left\|c_{h}-c\right\|_{1} \leq & \left(2 D+\text { Const }\left\|u_{h}\right\|_{1}\right) h\|c\|_{2}+L_{K} \text { Const }_{4}|p|_{1,4}\left\|c-c_{h}\right\|_{1} \\
& + \text { Const }_{5}\|c\|_{1}\left\|u-u_{h}\right\|_{1}+\text { Const }_{6}|K|_{\infty}\left\|p-p_{h}\right\|_{1} .
\end{aligned}
$$


Substituting (27) and (28) and using imbedding $H^{k} \hookrightarrow W^{k-1,6}, k=1,2$, we conclude that if the data are small in the sense of Theorem 1, namely, if

$D>\left(L_{K}\right.$ Const $_{4}$ Const $_{1}+\frac{\text { Const }_{5}}{\nu}$ Const $_{1}\|g\|_{0,3}+|K|_{\infty}$ Const $\left._{6}\|g\|_{0,3}\right)$,

then $\left\|c-c_{h}\right\|_{1}=O(h)$, whence, using (27) and (28) again, we obtain (21).

Remark 1 In view of approximation properties of $V_{h}$, it seems that the approximation error in $u_{h}$ is not optimal in $h$ (contrary to $\left\|p-p_{h}\right\|_{1}$ and $\left\|c-c_{h}\right\|_{1}$, which are of optimal order). However, there are chances that the actual order of approximation of the velocity may be improved, or, in case of more regular data, even restored to optimal level, since by (27) this error depends on a $c_{h}$ error in a norm which doesn't involve derivatives.

\section{UNIQUENESS OF APPROXIMATE SOLUTIONS}

We estimate the difference between two possibly different solutions $c_{1}, c_{2} \in \mathcal{M}$ for the same data. The ball $\mathcal{M}$ is defined as in Section 3 .

Using (26), (21) and estimates of discrete solutions to Stokes equations, we conclude that, for data and $h$ sufficiently small in the sense of Theorem 1 , the difference $\bar{c}=c_{1}-c_{2}$ satisfies $\|\bar{c}\|_{1} \leq 0$, so the solution must be unique.

\section{ACKNOWLEDGMENT}

I would like to thank Prof. M. Dryja for his valuable remarks and comments.

\section{REFERENCES}

Adams, R.A. (1975) Sobolev Spaces, Academic Press, New York.

Brezzi F., Fortin M. (1991) Mixed and Hybrid Finite Element Methods, Springer-Verlag, New York.

Bercovier, M. and Pironneau, O. (1979) Error estimates for Finite Element Method solution of the Stokes problem in the primitive variables, Num. Math., 33, 211-224.

Ciarlet, P.G. (1991) Basic Error Estimates for Elliptic Problems, Handbook of Numerical Analysis, vol II, Finite Element Methods (Part I), Elsevier Science Publishers B.V. (North-Holland).

Gilbarg, D. and Trudinger, N.S. (1983) Elliptic Partial Differential Equations of Second Order, Springer-Verlag, Berlin.

Girault, V. and Raviart, P.A. (1986) Finite Element Method for Navier-Stokes Equations. Theory and Algorithms, Springer-Verlag, Berlin Heidelberg New York.

Hackbush, W. (1992) Elliptic Differential Equations. Theory and Numerical Treatment, Springer-Verlag, Berlin Heidelberg.

Kellogg, R.B. and Osborn, J.E. (1976) A regularity result for the Stokes problem in a convex polygon, J. Funct. Anal., 21, 397-431. 
Krzyżanowski, P. (1994) On stationary flow of asymmetric fluids with diffusion, Math. Meth. Appli. Sci., 17 837-854.

Lukaszewicz, G. (1991) On diffusion in viscous fluids. Mixed boundary conditions, Proc. 'First European Conference on Elliptic and Parabolic Problems', Pont-a-Mousson, June 1991.

Petrosyan, L.G. (1984) Some Problems of Mechanics of Fluids with Antisymmetric Stress Tensor, Izd. Erev. Univ., Erevan (in Russian).

Popel, A.S., Regirer, S.A. and Usick, P.I. (1974) A continuum model of blood flow, Biorheology, XI, 427-437.

Temam, R. (1979) Navier - Stokes Equations. Theory and Numerical Analysis, NorthHolland, Amsterdam New York Oxford. 